\title{
The Relevance of Serum Cystatin C Level of Different Classification of Atrial Fibrillation
}

\author{
Yuqing Duan, Jianhui Xu, Wei Hu, Peng Li, Rui Li* \\ Department of Cardiology, The Central Hospital of Xiaogan, Wuhan University of Science and Technology, Wuhan, China \\ Email:dyqqh7@126.com, *chinaxglr@126.com
}

How to cite this paper: Duan, Y.Q., $\mathrm{Xu}$, J.H., Hu, W., Li, P. and Li, R. (2019) The Relevance of Serum Cystatin C Level of Different Classification of Atrial Fibrillation. International Journal of Clinical Medicine, 10, 404-412.

https://doi.org/10.4236/ijcm.2019.108033

Received: July 16, 2019

Accepted: August 13, 2019

Published: August 16, 2019

Copyright $\odot 2019$ by author(s) and Scientific Research Publishing Inc. This work is licensed under the Creative Commons Attribution International License (CC BY 4.0).

http://creativecommons.org/licenses/by/4.0/

\section{Abstract}

Objective: To investigate the relationship between serum level of cystatin C (Cys-C) and AF (atrial fibrillation) and its clinical classification. Method: From January 2017 to April 2019, 168 cases of Xiaogan Central Hospital were chosen as the object of this study. The subjects were divided into 86 patients with $\mathrm{AF}$ and 82 patients in the control group. The AF group was divided into paroxysmal AF group (29 cases), persistent AF group (27 cases) and permanent AF group (29 cases) according to the European atrial fibrillation management guidelines and the North America Society of Pacing and Electrophysiology (NASPE) arrhythmia group organized the categorization of AF. Results: Compared with the control group, the level of the serum Cys-C was significantly higher in the AF group, the difference was statistically significant $(P<0.05)$. There was significant difference in Cys-C level in patients with different types of AF $(P<0.05)$. The levels of neutrophil percentage, low density lipoprotein cholesterol (LDL-C), left ventricular diameter, left atrial diameter, $\mathrm{C}$-reactive protein (CRP) and homocysteine in the AF group were significantly higher than those in the control group $(P<0.05)$. The difference of neutrophil percentage, LDL-C, left ventricular ejection fraction, left atrial diameter, CRP and homocysteine levels in patients with different types of atrial fibrillation was statistically significant $(P<0.05)$. Logistic analysis showed that the serum Cys-C level, CRP, homocysteine, left ventricular diameter, left atrial diameter could be used as an independent predictor of atrial fibrillation when other factors were corrected. Conclusion: Serum Cys-C level in atrial fibrillation group is significantly higher than the control group, there are differences between different atrial fibrillation clinical classification, its level increased with duration of atrial fibrillation. Serum Cys-C level and inflammatory markers $\mathrm{CRP}, \mathrm{WBC}$ and neutrophilic granulocyte percentage were positively correlated, indicating that serum cystatin $\mathrm{C}$ is associated with chronic inflammation, involved in the occurrence of atrial fibrillation, maintain and recurrence. Logistic analysis showed that the serum cystatin $\mathrm{C}$ level could be 
used as an independent predictor of atrial fibrillation when other factors were corrected.

\section{Keywords}

Cystatin C, Atrial Fibrillation, Inflammatory

\section{Introduction}

Atrial fibrillation (AF) is a complex and dangerous arrhythmia. Serious disorder of atrial electrical activity, which is a regular and orderly electrical activity and replaced by rapid and disordered tremor, is the main cause of its occurrence. Epidemiological investigation shows that the prevalence of AF in Chinese is about $0.77 \%-2.8 \%$, accounting for about one-third of all hospitalized arrhythmia patients. The morbidity and prevalence of atrial fibrillation increased with age [1]. The main causes of death in patients with atrial fibrillation are progressive heart failure (HF), cardiac arrest and cerebral apoplexy [2]. Reasonable treatment in time can significantly reduce mortality in patients with atrial fibrillation. Therefore, how to diagnose atrial fibrillation as soon as possible and assess the patient's condition, so that these patients get treatment as soon as possible, to minimize the damage and harm caused by the disease, is still a hot topic in the medical field. Cystatin C (Cys-C) is a low-molecular-weight secretory protein of the human body. Almost all nucleated cells can produce this secreted protein, and the secretion process is rarely affected by factors such as gender, age, bilirubin, blood lipids, muscle mass, etc., so the serum Cys-C production rate and serum level are relatively constant. In recent years, it has been found that serum Cys- $\mathrm{C}$ is more sensitive than creatinine ( $\mathrm{Cr}$ ) and Serum urinary nitrogen (BUN) as an indicator of glomerular filtration rate. It is currently used as a sensitive indicator for assessing early damage to renal function [3] [4]. In 2010, Deo et al. showed that serum Cys-C level was positively correlated with the prevalence of atrial fibrillation. It was concluded that renal dysfunction, which was evaluated by serum Cys-C, was an independent predictor of AF prevalence [5]. In recent years, Cys $\mathrm{C}$ has been found to be able to predict new or worsening cardiovascular diseases [6]. In addition, studies have shown that higher levels of serum Cys-C may increase the risk of cardiovascular autonomic nervous dysfunction [7]. At present, many literatures and experiments have proved that elevated serum Cys- $\mathrm{C}$ is an independent risk factor for atrial fibrillation [8]. However, whether there is a correlation between serum Cys-C level and types of atrial fibrillation still needs to be further explored. The purpose of this study was to explore the relationship between cystatin $\mathrm{C}$ and atrial fibrillation types, and to provide a new idea for early clinical evaluation of atrial fibrillation types.

\section{Date and Methods}

1) Clinical Data: 86 patients with non-valvular atrial fibrillation who were 
admitted to Xiaogan Central Hospital from January 2017 to April 2019. According to the European Society of Cardiovascular Disease (ESC), the North American Pacing and Electrophysiology Society (NASPE), the above atrial fibrillation patients were divided into three subgroups, paroxysmal AF (29 cases). People, accounting for 33.72\%; persistent AF (27 cases), accounting for 31.39\%; permanent AF (30 cases), accounting for $34.88 \%$. At the same time, 82 patients in the normal control group were selected, and the general situation (gender, age, creatinine, cystatin- $C$, etc.) matched the atrial fibrillation group and the electrocardiogram was normal sinus rhythm. Inclusion criteria: Atrial fibrillation can be diagnosed according to the surface electrocardiogram (ECG) or 24-hour Holter, the patients' ages were 18 - 99 years, male or female. Exclusion criteria: patients with congenital heart disease, rheumatic heart disease, and prosthetic valve replacement or repair; history of coronary artery bypass grafting or coronary stent implantation; acute coronary syndrome (ACS) within 3 months of onset; patients with clear hypertension, diabetes, blood disease, thyroid dysfunction, liver and kidney dysfunction, autoimmune diseases, tumor diseases, nervous system diseases; patients with acute and chronic infectious diseases; patients with trauma, surgical history and anti-infective drugs in the recent 30 days; New York Heart Association Cardiac Function Grade 3 or more; patients with statins lipid-lowering drugs in the recent 30 days.

2) Methods Record: The patient's general information, gender, age, atrial fibrillation type, CHA2DS2VASc score, HAS-BLED score, the peripheral venous blood was collected on an empty stomach the next morning, blood routine, liver function, renal function, electrolytes, blood lipids, blood homocysteine, high-sensitivity C-reactive protein, etc. Early cardiac color Doppler ultrasound examination, measurement of left atrial diameter (LAD), left ventricular end-diastolic diameter (LVDD), left ventricular ejection fraction (LVEF).

3) Statistical Analysis: Statistical analysis was carried out by SPSS 23.0 software. The comparison between the two groups of measurement data subject to normal distribution was carried out by T-test; the comparison of more than three groups was carried out by one-way ANOVA test, and the comparison between groups was carried out by $\mathrm{T}$-test. The measurement data not subject to normal distribution was carried out by Wilcoxon rank sum test of two independent samples; and the counting data was analyzed by $\chi^{2}$ test. For comparison of multi-group measurement data, variance analysis was used for normal distribution and homogeneous variance, Kruskal-Wallis $\mathrm{H}$ test was used for non-normal distribution or uneven variance. Logistic regression analysis was used to analyze the risk factors of atrial fibrillation $(P<0.05)$.

\section{Results}

\subsection{Comparison of Clinical Data between Atrial Fibrillation Group and Control Group}

The general information of the patients in the two groups is shown in Table 1. 
Table 1. Comparison of clinical data between atrial fibrillation group and control group.

\begin{tabular}{|c|c|c|c|c|}
\hline Related indicators & $\begin{array}{l}\text { Control group } \\
\quad(\mathrm{n}=82)\end{array}$ & $\begin{array}{c}\text { AF group } \\
(\mathrm{n}=86)\end{array}$ & Statistics & $P$ \\
\hline age & $69.55 \pm 10.19$ & $71.40 \pm 11.21$ & $\mathrm{t}=1.901$ & $P=0.063$ \\
\hline WBC count $\left(10^{9} / 1\right)$ & $6.29 \pm 3.56$ & $6.31 \pm 2.48$ & $\mathrm{t}=0.109$ & $P=0.929$ \\
\hline Neutrophil percentage (\%) & $64.25 \pm 8.92$ & $69.33 \pm 9.03$ & $\mathrm{t}=3.271$ & $P=0.002$ \\
\hline Creatinine $(\mathrm{mmoI} / \mathrm{L})$ & $84.52 \pm 10.23$ & $82.33 \pm 11.96$ & $\mathrm{t}=1.505$ & $P=0.139$ \\
\hline Total cholesterol $(\mathrm{mmol} / \mathrm{L})$ & $3.48 \pm 1.01$ & $3.53 \pm 0.91$ & $\mathrm{t}=1.033$ & $P=0.501$ \\
\hline $\mathrm{LDL}-\mathrm{C}(\mathrm{mmol} / \mathrm{L})$ & $1.23 \pm 0.55$ & $1.66 \pm 0.62$ & $\mathrm{t}=2.880$ & $P=0.006$ \\
\hline HDL-C (mmol/L) & $1.16 \pm 0.54$ & $1.18 \pm 0.29$ & $t=0.647$ & $P=0.224$ \\
\hline Triglyceride $(\mathrm{mmol} / \mathrm{L})$ & $1.32 \pm 0.56$ & $1.28 \pm 0.83$ & $t=0.561$ & $P=0.576$ \\
\hline $\operatorname{LVEF}(\%)$ & $60.22 \pm 7.48$ & $53.93 \pm 8.14$ & $\mathrm{t}=2.133$ & $P=0.004$ \\
\hline Left inner diameter $(\mathrm{cm})$ & $4.02 \pm 1.23$ & $4.70 \pm 0.66$ & $\mathrm{t}=2.544$ & $P=0.014$ \\
\hline Left atrial diameter $(\mathrm{cm})$ & $3.14 \pm 1.18$ & $4.34 \pm 0.77$ & $\mathrm{t}=2.976$ & $P<0.001$ \\
\hline $\mathrm{CRP}(\mathrm{mg} / \mathrm{L})$ & $2.05 \pm 10.98$ & $6.93 \pm 17.76$ & $t=2.23$ & $P=0.004$ \\
\hline Homocysteine $(\mathrm{mmol} / \mathrm{L})$ & $7.96 \pm 5.21$ & $13.96 \pm 3.40$ & $\mathrm{t}=3.79$ & $P<0.001$ \\
\hline Cystatin C (mg/L) & $0.67 \pm 0.98$ & $0.92 \pm 0.39$ & $\mathrm{t}=4.150$ & $P<0.001$ \\
\hline
\end{tabular}

WBC count $=$ White blood cell count LDL-C = Low density lipoprotein cholesterol; HDL-C = High density lipoprotein cholesterol; LVEF Left ventricular ejection fraction.

Compared with the control group, the average age of the control group was $69.55 \pm 10.19$ years, and that of the atrial fibrillation group was $71.40 \pm 11.21$ years. The percentage of neutrophils, LDL-C, left ventricular diameter, left atrial diameter, $\mathrm{CRP}, \mathrm{HCY}$ and serum Cys-C levels in the atrial fibrillation group were higher than the control group, the difference was statistically significant $(P<$ $0.05)$. The left ventricular ejection fraction of the atrial fibrillation group was smaller than that of the control group, and the difference was statistically significant $(P<0.05)$. There was no significant difference in white blood cell count, creatinine, total cholesterol, high density lipoprotein cholesterol and triglyceride between the two groups $(P>0.05)$.

\subsection{Comparison of Clinical Data between Different Atrial Fibrillation Groups}

The average age of patients with paroxysmal atrial fibrillation was $67.45 \pm 12.25$, the average age of patients with persistent atrial fibrillation was $66.92 \pm 9.88$ years, and the average age of patients with permanent atrial fibrillation was $76.67 \pm$ 9.15 years old. There was no significant difference in the age of patients with different types of atrial fibrillation $(\mathrm{F}=5.018, P=0.212)$. The serum Cys-C levels in the permanent $\mathrm{AF}$ were higher than in the persistent $\mathrm{AF}$, and the persistent $\mathrm{AF}$ was higher than the paroxysmal AF. All were statistically significant $(P<$ $0.05)$. At the same time, the study found that the difference in the percentage of neutrophils, LDL-C, left atrial diameter, LVEF, CRP, HCY in different types of patients were statistically significant $(P<0.05)$, of which, for neutrophils Per- 
centage, LDL-C, left atrial diameter, CRP, HCY, permanent AF were higher than paroxysmal AF and persistent AF, the difference was statistically significant $(P<$ 0.05). For left ventricular ejection fraction, the permanent AF was lower than paroxysmal AF and persistent AF, the difference was statistically significant $(P<$ 0.05). There were no significant differences in white blood cell count, creatinine, total cholesterol, high-density lipoprotein cholesterol, and triglycerides between patients with different types of atrial fibrillation $(P>0.05)$ (Table 2).

\subsection{Logistic Multivariate Analysis}

As shown in Table 3, univariate logistic regression analysis was performed on factors such as age, neutrophil percentage, LDL-C, plasma homocysteine, CRP, and serum Cys- $\mathrm{C}$, and then multivariate analysis was performed on single-factor meaningful variables. It is shown that serum Cys-C, CRP, homocysteine, cystatin $\mathrm{C}$, left ventricular diameter, and left atrial diameter are risk factors for atrial fibrillation.

\section{Discussion}

Atrial fibrillation is a disease that increases mortality. Some studies have shown that atrial fibrillation can increase the risk of sudden cardiac death, major cardiovascular adverse events, heart failure, ischemic heart disease, cerebral infarction, peripheral artery disease, chronic kidney disease and other related diseases [9]. Current studies suggest that atrial fibrillation is mainly associated with atrial remodeling, electrical remodeling, autonomic nervous system function, gene mutation, increased activity of renin-angiotensin-aldosterone system and

Table 2. Comparison of clinical data between different atrial fibrillation groups.

\begin{tabular}{|c|c|c|c|c|c|}
\hline Related indicators & $\begin{array}{l}\text { Paroxysmal AF } \\
\qquad(\mathrm{n}=29)\end{array}$ & $\begin{array}{l}\text { Persistent AF } \\
\quad(\mathrm{n}=27)\end{array}$ & $\begin{array}{l}\text { Permanent AF } \\
\quad(\mathrm{n}=30)\end{array}$ & Statistics & $P$ \\
\hline Age & $67.45 \pm 12.25$ & $66.92 \pm 9.88$ & $76.67 \pm 9.15$ & $\mathrm{~F}=5.018$ & $P=0.212$ \\
\hline WBC count $\left(10^{9} / 1\right)$ & $6.03 \pm 1.71$ & $6.98 \pm 3.29$ & $7.01 \pm 2.22$ & $F=0.668$ & $P=0.518$ \\
\hline Neutrophil percentage (\%) & $68.45 \pm 9.66$ & $70.71 \pm 10.44$ & $74.88 \pm 7.91$ & $\mathrm{H}=10.033$ & $P=0.004$ \\
\hline Creatinine $(\mathrm{mmoI} / \mathrm{L})$ & $77.77 \pm 10.93$ & $80.9 \pm 9.47$ & $82.15 \pm 13.45$ & $\mathrm{H}=2.838$ & $P=0.242$ \\
\hline $\begin{array}{l}\text { Total cholesterol } \\
(\mathrm{mmol} / \mathrm{L})\end{array}$ & $3.67 \pm 0.83$ & $3.85 \pm 0.95$ & $3.21 \pm 0.87$ & $\mathrm{~F}=2.092$ & $P=0.137$ \\
\hline LDL-C (mmol/L) & $1.52 \pm 0.48$ & $1.88 \pm 0.67$ & $2.01 \pm 0.66$ & $\mathrm{~F}=8.230$ & $P=0.003$ \\
\hline $\mathrm{HDL}-\mathrm{C}(\mathrm{mmol} / \mathrm{L})$ & $1.25 \pm 0.27$ & $1.26 \pm 0.28$ & $1.07 \pm 0.30$ & $\mathrm{~F}=2.098$ & $P=0.136$ \\
\hline Triglyceride (mmol/L) & $1.59 \pm 1.25$ & $1.25 \pm 0.72$ & $1.10 \pm 0.54$ & $\mathrm{~F}=1.198$ & $P=0.313$ \\
\hline LVEF (\%) & $57.45 \pm 3.64$ & $55.85 \pm 8.35$ & $51.29 \pm 0.95$ & $\mathrm{~F}=7.555$ & $P=0.004$ \\
\hline Left inner diameter $(\mathrm{cm})$ & $4.51 \pm 0.58$ & $4.68 \pm 0.75$ & $4.84 \pm 0.64$ & $\mathrm{~F}=0.845$ & $P=0.437$ \\
\hline Left atrial diameter $(\mathrm{cm})$ & $3.82 \pm 0.77$ & $4.42 \pm 0.71$ & $4.61 \pm 0.68$ & $\mathrm{~F}=10.265$ & $P=0.001$ \\
\hline CRP (mg/L) & $1.16 \pm 1.98$ & $2.02 \pm 1.05$ & $14 \pm 25.1$ & $H=26.236$ & $P=0.001$ \\
\hline Homocysteine (mmol/L) & $16.7 \pm 3.81$ & $17.3 \pm 1.93$ & $21.22 \pm 3.79$ & $\mathrm{~F}=15.912$ & $P<0.001$ \\
\hline Cystatin C (mg/L) & $0.65 \pm 0.09$ & $0.82 \pm 0.27$ & $1.15 \pm 0.45$ & $\mathrm{H}=34.332$ & $P<0.001$ \\
\hline
\end{tabular}


Table 3. Logistic multivariate analysis results.

\begin{tabular}{lcccccc}
\hline Influencing factor & $\mathrm{B}$ & S.E. & Wald & $P$-value & OR & $95 \% \mathrm{CI}$ \\
\hline Cystatin c & 2.668 & 0.256 & 7.986 & 0.004 & 12.56 & $0.132-49.895$ \\
$\begin{array}{l}\text { Neutrophil } \\
\text { percentage }\end{array}$ & 1.180 & 2.75 & 0.768 & 0.108 & 2.75 & $0.654-10.411$ \\
LDL-C & 0.120 & 0.346 & 0.059 & 0.988 & 1.601 & $1.447-1.768$ \\
CRP & 0.125 & 0.028 & 18.186 & 0.000 & 1.522 & $1.144-1.965$ \\
Homocysteine & 0.134 & 0.036 & 15.981 & 0.000 & 1.301 & $1.159-1.464$ \\
LVEF & -0.049 & 0.038 & 0.965 & 0.213 & 0.951 & $0.793-1.128$ \\
Left inner diameter & 0.137 & 0.044 & 6.175 & 0.010 & 1.215 & $1.052-1.317$ \\
Left atrial diameter & 0.104 & 0.022 & 12.358 & 0.001 & 1.098 & $1.051-1.181$ \\
\hline
\end{tabular}

inflammatory reaction [10]. Inflammatory reaction was found to be closely related to the occurrence and progression of atrial fibrillation. Inflammatory factors such as interleukin (IL) [11], C-reactive protein (CRP) [12], tumor necrosis factor- $\alpha$ (TNF- $\alpha$ ) [13] were found to be associated with atrial fibrillation. Cysteine protease inhibitor $\mathrm{C}(\mathrm{Cys}-\mathrm{C})$ is a low molecular weight $(13 \mathrm{kDa})$ protein produced by all nucleated cells. More and more studies have confirmed that Cys- $\mathrm{C}$ may play a key role in the detection and evaluation of cardiovascular diseases in addition to being an indicator of impaired renal function. Wang et al. have shown that higher levels of serum Cys-C are independently associated with increased risk of cardiovascular events, and this risk is not significantly associated with GFR [14]. Cys C is also released from cardiac myocytes, and hypoxia increases its production [15]. Increased serum Cys-C level can inhibit the effect of protease and lead to remodeling of vascular wall. At the same time, Cys-C can reduce the decomposition of atrial fibroblasts and cause myocardial fibrosis. Myocardial fibrosis is the pathological basis of the occurrence and progression of atrial fibrillation [16]. HCY is an independent predictor of cardiovascular and cerebrovascular diseases [17]. Serum Cys-C can inhibit the activity of cysteine protease and reduce the decomposition of $\mathrm{HCY}$, damage vascular endothelium and inhibit the synthesis of NO. Both of them participate in the oxidative stress process, lead to ischemia and hypoxia, and cause changes in atrial structure. It is easy to induce atrial fibrillation [18]. Cys-C is involved in many inflammatory reactions. Serum Cys-C and its degradation products can directly affect the migration, chemotaxis and phagocytosis of neutrophils, thus participating in the whole inflammatory process [19]. Inflammatory reaction leads to fibrosis of atrial myocytes, which leads to atrial enlargement, and finally changes of atrial structure, leading to atrial fibrillation. Korantzopoulos et al. found that inflammation contributes to electrical remodeling of atrial fibrillation, especially in the recurrence or evolution of atrial fibrillation into permanent atrial fibrillation [20], which is consistent with the results of Samouilidou's study [21]. In Buddha's Framingham's study [22], we found a close correlation between LAD and 
atrial fibrillation: the prevalence of atrial fibrillation increased with the increase of LAD. Wang Xin and other studies indicated that high serum Cys-C level was a risk factor for left atrial dilatation [23], and electrical remodeling caused by atrial dilatation and fibrosis could trigger atrial fibrillation [24]. High Cys-C level is a highly sensitive marker of mild renal insufficiency. Mild renal insufficiency is associated with microvascular endothelial insufficiency [25]. High Cys-C level may lead to microvascular endothelial insufficiency, activate RAAS system, accumulate extracellular matrix, accelerate atrial fibrosis, and activation of RAAS system may also lead to renal function damaged.

In this study, we found that serum Cys-C level in AF group was significantly higher than that in non-AF group. There were differences among clinical types of AF. The level of Cys-C increased with the duration of AF $(P<0.05)$. In the process of analyzing the correlation between Cys- $\mathrm{C}$ and various clinical indicators, we found that serum Cys-C and inflammation were associated with AF. Indicators such as hypersensitivity protein, white blood cell count and percentage of neutrophils were positively correlated, which supported that the occurrence and development of atrial fibrillation was closely related to inflammation. At the same time, the level of LDL-C in atrial fibrillation group was higher than that in non-atrial fibrillation group, and increased with the prolongation of atrial fibrillation time $(P<0.05)$. In multivariate logistic regression analysis, we found that left ventricular diameter can be a risk factor for atrial fibrillation. This may be because atrial fibrillation can lead to ventricular fibrosis, enlargement of ventricular diameter, decrease of left ventricular ejection fraction, and decrease of left ventricular ejection fraction, which can also promote atrial enlargement. They form a vicious circle. Logistic analysis showed that the elevated serum cystatin $\mathrm{C}$ level could be used as an independent predictor of atrial fibrillation after adjusting for other factors.

Because the measurement of serum Cys- $\mathrm{C}$ is simple, inexpensive and painful, and it can also be used as an evaluation index of renal function, serum Cys- $\mathrm{C}$ is expected to be an effective marker for evaluating the occurrence, maintenance and recurrence of atrial fibrillation, and has important clinical significance. Serum Cys- $C$ is also an index closely related to renal function, which is of great significance for research. It is also important to investigate and analyze the relationship between kidney disease and cardiovascular disease. However, this study is a retrospective analysis. Although many factors that may affect the results of the study have been excluded, due to the small sample size and the drug (the patient may take long-term medications such as statins and other cardiovascular diseases before admission) and various factors, the persuasiveness of the research results is limited, and the reliability of the final conclusion still needs to be further confirmed by large-scale and more rigorous experimental analysis. The influence of many factors, the persuasiveness of the research results is limited, and the reliability of the final conclusion still needs to be further confirmed by large-scale and more rigorous experimental analysis. 


\section{Conflicts of Interest}

The authors report no relationships that could be construed as a conflict of interest.

\section{References}

[1] Chugh, S.S., Havmoeller, R., Narayanan, K., et al. (2014) Worldwide Epidemiology of Atrial Fibrillation: A Global Burden of Disease 2010 Study. Circulation, 129, 837-847. https://doi.org/10.1161/CIRCULATIONAHA.113.005119

[2] Kirchhof, P., Benussi, S., Kotecha, D., et al. (2016) ESC Guidelines for the Management of Atrial Fibrillation Developed in Collaboration with EACTS. European Heart Journal, 37, 2893-2962. https://doi.org/10.1093/eurheartj/ehw210

[3] Ferguson, T.W., Komenda, P. and Tangri, N. (2015) Cystatin C as a Biomarker for Estimating Glomerular Filtration Rate. Current Opinion in Nephrology and Hypertension, 24, 295-300. https://doi.org/10.1097/MNH.0000000000000115

[4] Inker, L.A., Schmid, C.H., Tighiouart, H., et al. (2012) Estimating Glomerular Filtration Rate from Serum Creatinine and Cystatin C. New England Journal of Medicine, 367, 20-29. https://doi.org/10.1056/NEJMoa1114248

[5] Deo, R., Katz, R., Kestenbaum, B., et al. (2010) Impaired Kidney Function and Atrial Fibrillation in Elderly Subjects. Journal of Cardiac Failure, 16, 55-60. https://doi.org/10.1016/j.cardfail.2009.07.002

[6] Guerchicoff, A., Stone, G.W., Mehran, R., et al. (2015) Analysis of Biomarkers for Risk of Acute Kidney Injury after Primary Angioplasty for Acute ST-Segment Elevation Myocardial Infarction: Results of the HORIZONS-AMI Trial. Catheterization Cardiovascular Intervention, 585, 335-342. https://doi.org/10.1002/ccd.25620

[7] Yang, X., Lin, Q., Li, X., Wu, L., Xu, W., Zhu, Y., Yao, B., et al. (2019) Cystatin C Is an Important Biomarker for Cardiovascular Autonomic Dysfunction in Chinese Type 2 Diabetic Patients. Journal of Diabetes Research, 2019, Article ID: 1706964. https://doi.org/10.1155/2019/1706964

[8] Peng, L. and Fang, Z. (2015) Expression and Significance of Serum Heat Shock Protein 70 and Cystatin $\mathrm{C}$ in Elderly Patients with Chronic Atrial Fibrillation. Hebei Medical College, No. 9, 1455-1458.

[9] Khaji, A., Kowey, P.R., et al. (2016) Update on Atrial Fibrillation. Texas Heart Institute Journal, 43, 412-414. https://doi.org/10.14503/THIJ-16-5916

[10] Huang, C., Zhang, S., Huang, D., et al. (2018) Atrial Fibrillation: Current Understanding and Treatment Recommendations-2018. Chinese Journal of Cardiac Pacing and Electrophysiology, 32, 315-368.

[11] Marcus, G.M., Smith, L.M., Ordovas, K., et al. (2010) Intracardiac and Extracardiac Markers of Inflammation during Atrial Fibrillation. Heart Rhythm, 7, 149-154. https://doi.org/10.1016/j.hrthm.2009.10.004

[12] Psychari, S.N., Apostolou, T.S., Sinos, L., et al. (2005) Relation of Elevated C-Reactive Protein and Interleukin-6 Levels to Left Atrial Size and Duration of Episodes in Patients with Atrial Fibrillation. American Journal of Cardiology, 95, 764-767. https://doi.org/10.1016/j.amjcard.2004.11.032

[13] Qu, Y.C., Du, Y.M., Wu, S.L., et al. (2009) Activated Nuclear Factor-kappaB and Increased Tumor Necrosis Factor-Alpha in Atrial Tissue of Atrial Fibrillation. Scandinavian Cardiovascular Journal, 43, 292-297. https://doi.org/10.1080/14017430802651803 
[14] Wang, J., Sim, A.S., Wang, X.L., et al. (2008) Relations between Markers of Renal Function, Coronary Risk Factors and the Occurrence and Severity of Coronary Artery Disease. Atherosclerosis, 197, 853-859. https://doi.org/10.1016/j.atherosclerosis.2007.07.034

[15] Akgul, O., et al. (2013) Predictive Value of Elevated Cystatin C in Patients Undergoing Primary Angioplasty for ST-Elevation Myocardial Infarction. Journal of Critical Care, 28, 13-20. https://doi.org/10.1016/j.jcrc.2013.03.004

[16] Inker, L.A., Schmid, C.H., Tighiouart, H., et al. (2012) Estimating Glomerular Filtration Rate from Scrum Creatinine and Cystatin C. New England Journal of Medicine, 367, 20-29. https://doi.org/10.1056/NEJMoa1114248

[17] Chen, T., Chen, Y., Wang, Y., et al. (2013) Clinical Application of Plasma Cystatin C, Homocysteine, D-Dimer and Hypersensitive C-Reactive Protein in the Diagnosis and Treatment of Acute Cerebral Infarction. International Journal of Laboratory Medicine, No. 23, 3168-3169+3171.

[18] Zhang, R. (2014) Analysis of Correlation between Plasma Cystatin C and Homocysteine Levels and Carotid Atherosclerosis in Patients with Acute Cerebral Infarction. Journal of Practical Cardiocerebral Pulmonary Vascular Disease, No. 10, 28-30.

[19] Bengtsson, E., To, F., Grubb, A., et al. (2005) Absence of the Protease Inhibitor Cystatin C in Inflammatory Cells Results in Larger Plaque Area in Plaque Regression of apoE-Deficient Mice. Atherosclerosis, 180, 45-53. https://doi.org/10.1016/j.atherosclerosis.2004.12.025

[20] Korantzopoulos, P., Kolettis, T., Siogas, K., et al. (2003) Atrial Fibrination and Electrical Remodeling I the Potential Role of Inflammation and Oxidative Stress. Medical Science Monitor, 9, 226-229.

[21] Samouilidou, E.C. and Grapsa, E. (2008) Relationship of Serum Cystatin C with C-Reactive Protein and Apolipoprotein A1 in Patients on Hemodialysis. Renal Failure, 30, 711-715. https://doi.org/10.1080/08860220802212973

[22] Pearce, E.N., Yang, Q., Benjamin, E.J., et al. (2010) Thyroid Function and Left Ventricular Structure and Function in the Framingham Heart Study. Thyriod, 20, 369-373. https://doi.org/10.1089/thy.2009.0272

[23] Wang, X. (2012) Study on the Relationship between Serum Levels of Cystatin C and $\beta-2$ Microglobulin and Left Ventricular Structure and Function in Patients with Coronary Heart Disease. Shandong University, Jinan.

[24] Miller, L.M., Sood, M.M., Sood, A.R., et al. (2010) Cardiovascular Disease in End-Stage Renal Disease: The Challenge of Assessing and Managing Cardiac Disease in Dialysis Patients. International Urology and Nephrology, 42, 1007-1014. https://doi.org/10.1007/s11255-010-9857-x

[25] Alhusseiny, A.H., Al-Nimer, M.S. and Al-Neamy, S.I. (2015) Assessment of Serum Cystatin C Levels in Newly Diagnosed Acute Myocardial Infarction at the Onset and at the Time of Hospital Discharge. Cardiology Research, 6, 226-231. https://doi.org/10.14740/cr377w 